\title{
Amorphous $\mathrm{CoMoO}_{4}$ with Nanoporous Structure for Electrochemical Ammonia Synthesis under Ambient Conditions
}

\author{
Lin Xiao ${ }^{a}$, Yanqin Liang ${ }^{a, b}$, Zhaoyang Li ${ }^{a, b}$, Shuilin $W u^{a, b}$, Shengli Zhu ${ }^{a, b, d,}$, \\ Shuiyuan Luo ${ }^{d}$, Chuntao Chang, ${ }^{e}$, Zhenduo Cui ${ }^{a, b, ~ * ~}$
}

a School of Materials Science and Engineering, Tianjin University, Tianjin, 300350, China

b Key Laboratory of Advanced Ceramics and Machining Technology, Ministry of Education, Tianjin, 300350, China

c School of Materials Science and Engineering, Lanzhou Jiaotong University, Lanzhou, 730070, China

d College of Chemistry Engineering and Materials Science, Quanzhou Normal University, Quanzhou, 362000, Fujian, China

e School of Mechanical Engineering, Dongguan University of Technology, Dongguan, 523808, China

*Corresponding Authors.

E-mail address: slzhu@tju.edu.cn.changct@dgut.edu.cn.zdcui@tju.edu.cn

Number of pages: 9

Number of figures: 14

Number of tables: 1 


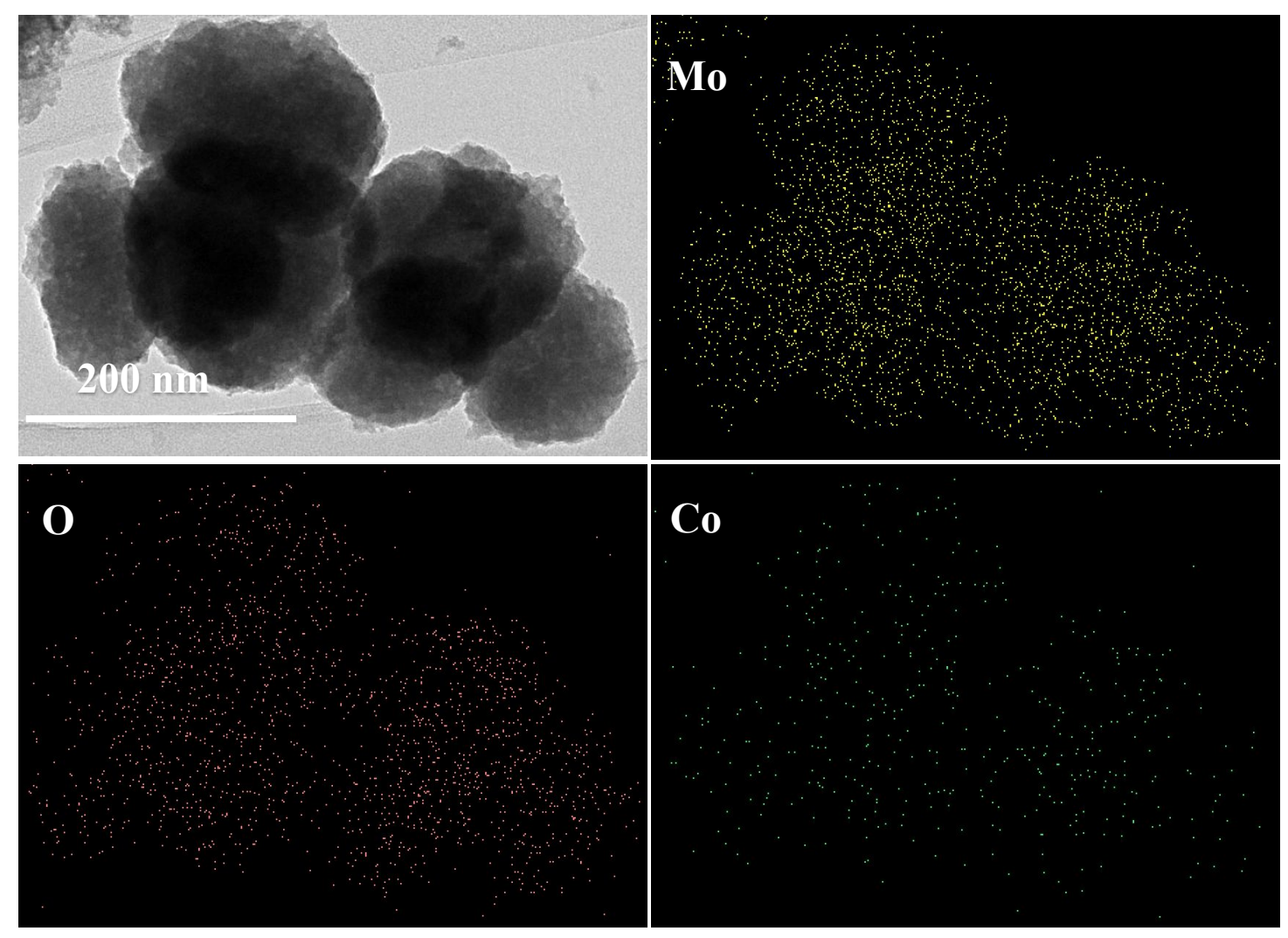

Figure S1. EDX element mapping images of $\mathrm{CoMoO}_{4}$.
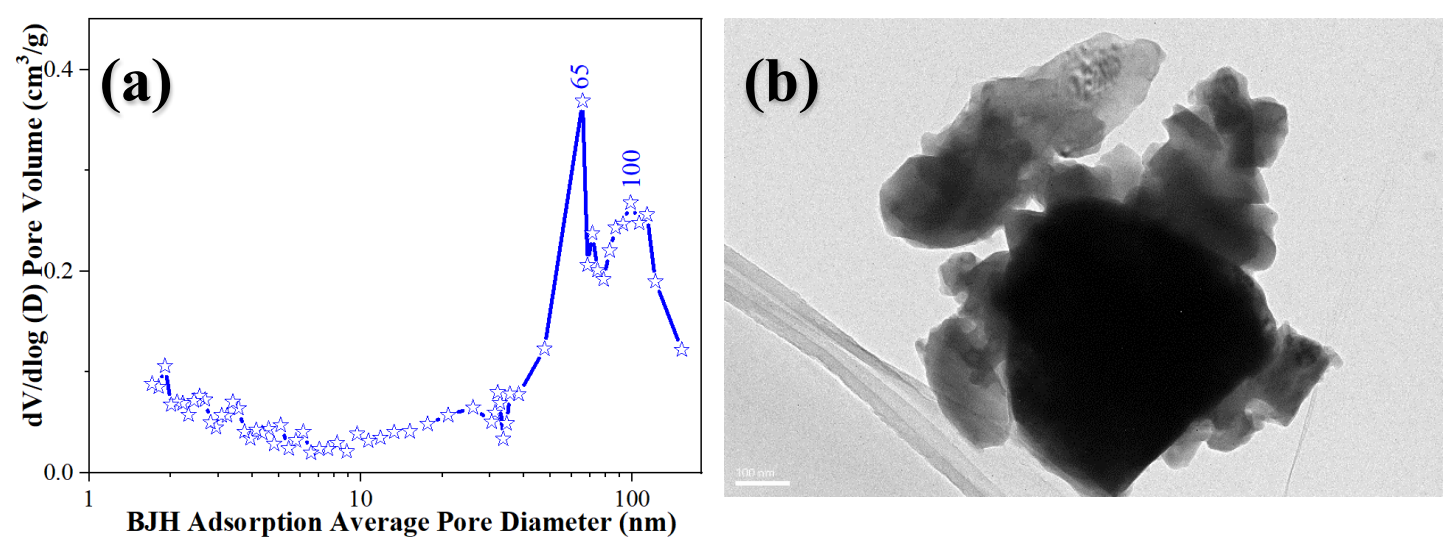

Figure S2. (a) BJH adsorption average pore diameter of amorphous $\mathrm{CoMoO}_{4}$ catalyst.

(b)TEM image of crystal $\mathrm{CoMoO}_{4}$ catalyst. 

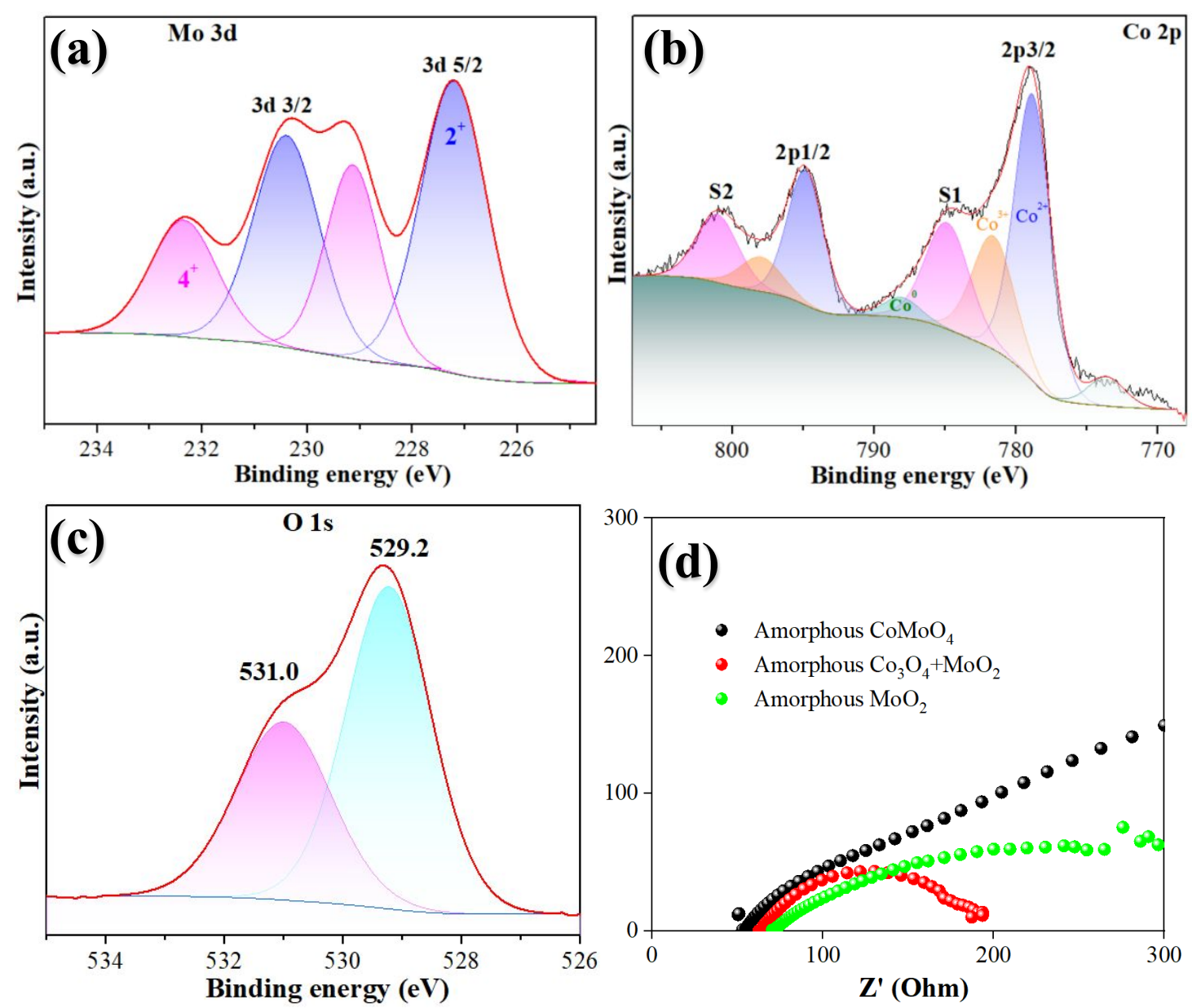

Figure S3. XPS spectra of mixture $\left(\mathrm{MoO}_{2}+\mathrm{Co}_{3} \mathrm{O}_{4}\right)$ for (a) Mo 3d and (b) $\mathrm{Co} 2 \mathrm{p}$ (c)

O 1s, (d) Nyquist plots of amorphous $\mathrm{CoMoO}_{4}$, amorphous $\mathrm{Co}_{3} \mathrm{O}_{4}+\mathrm{MoO}_{2}$ and amorphous $\mathrm{MoO}_{2}$. 


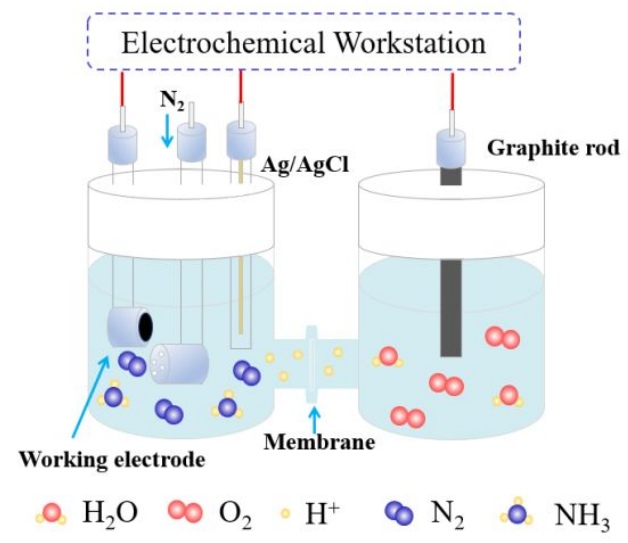

Figure S4. The schematic of electrolytic cell.
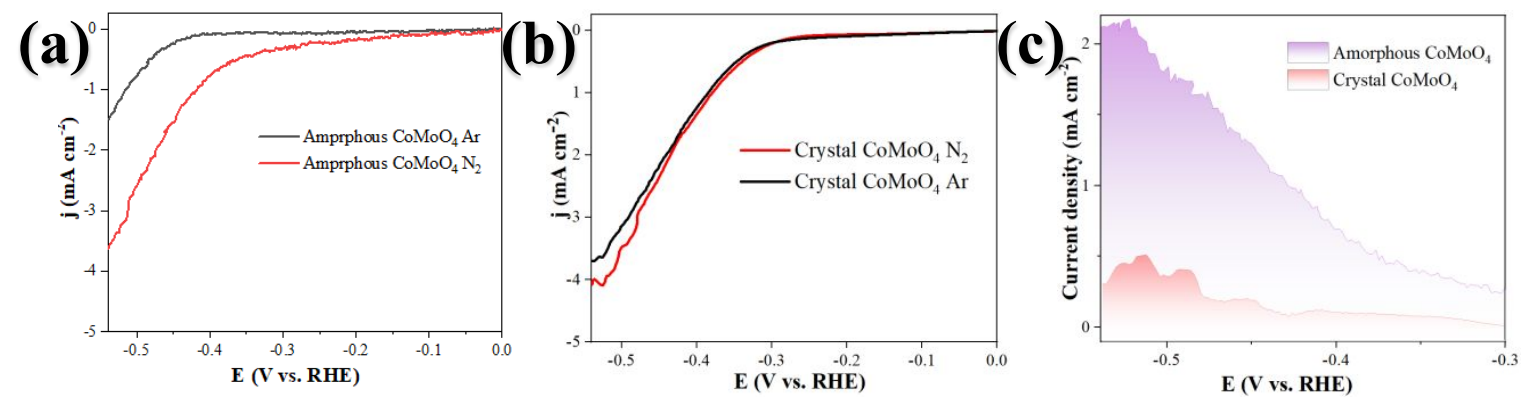

Figure S5. LSV curves of (a) amorphous $\mathrm{CoMoO}_{4}$ and (b) crystal $\mathrm{CoMoO}_{4}$ under $\mathrm{N}_{2}$ and $\mathrm{Ar},(\mathrm{c})$ Net current density.
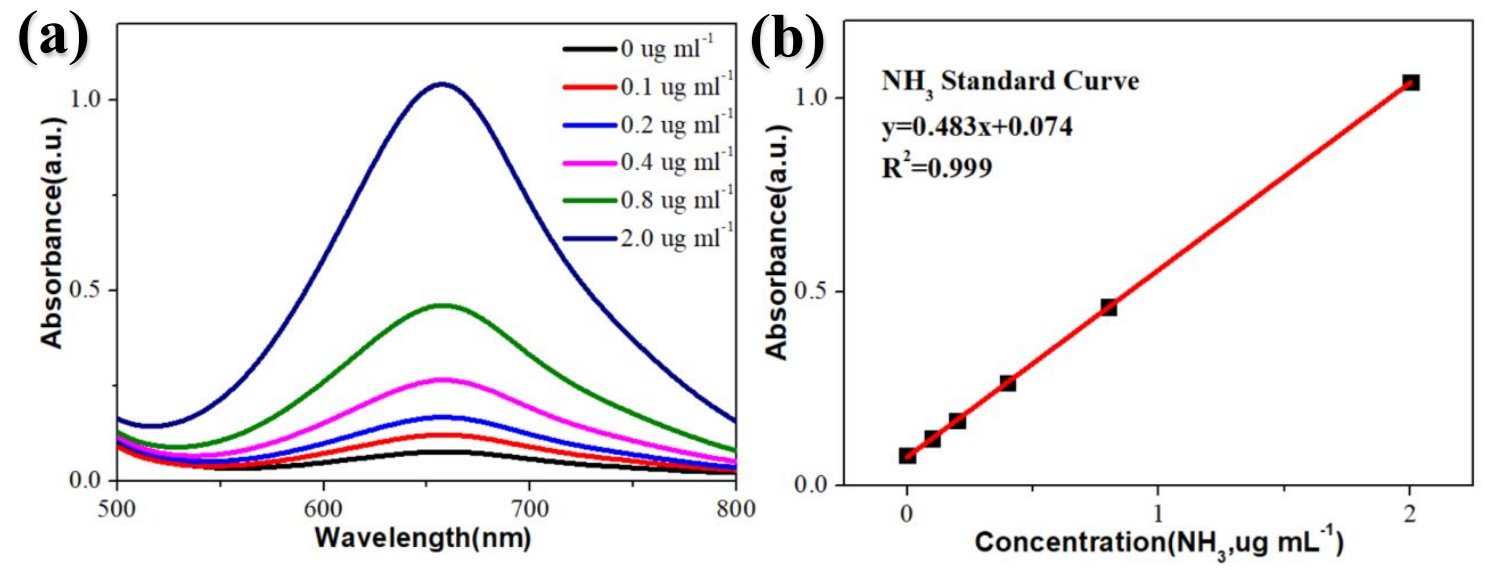

Figure S6. (a) UV-Vis spectrum of different $\mathrm{NH}_{4}{ }^{+}$content by indophenol assays after incubated for $2 \mathrm{~h}$ at room temperature. (b) Calibration curve used for the estimation of $\mathrm{NH}_{4}{ }^{+}$concentration. 

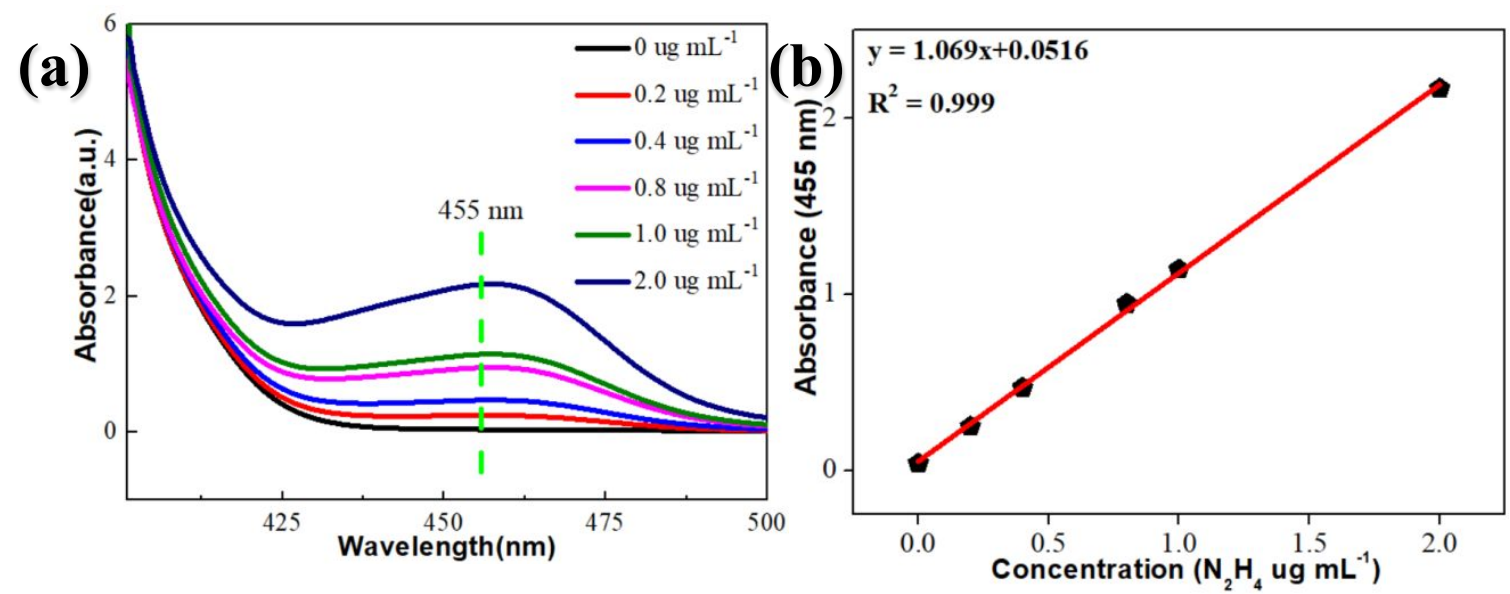

Figure S7. (a) UV-Vis spectra of different $\mathrm{N}_{2} \mathrm{H}_{4}$ content after incubated for $15 \mathrm{~min}$ at room temperature. (b) Calibration curve used for calculation of $\mathrm{N}_{2} \mathrm{H}_{4}$ concentration.

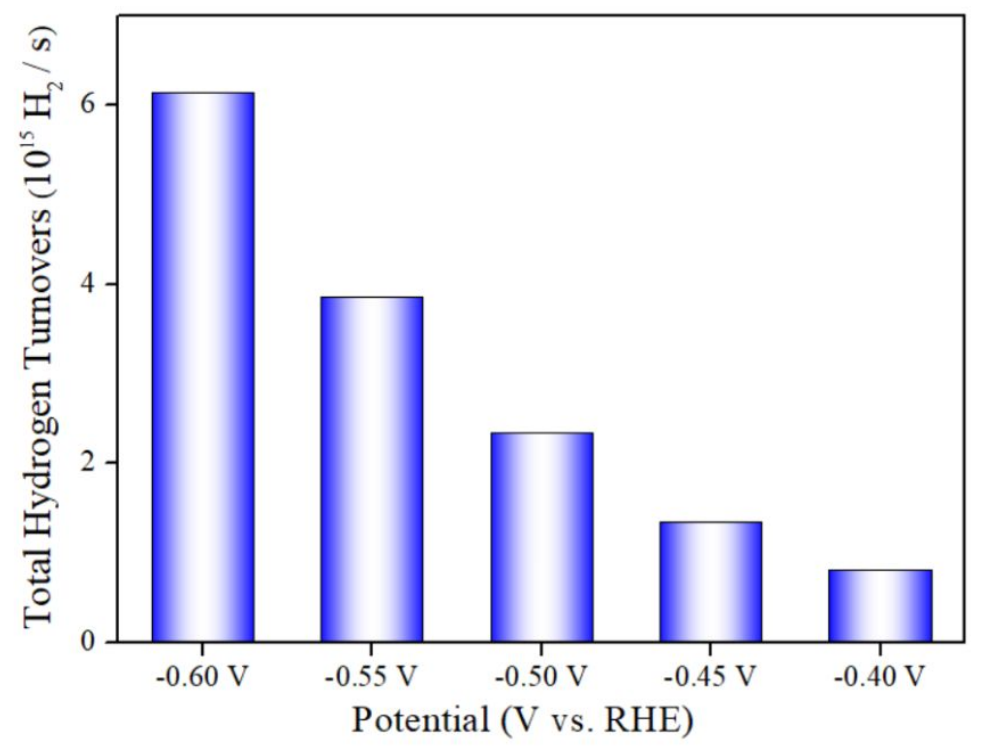

Figure S8. The total hydrogen turnovers at each given potential. 


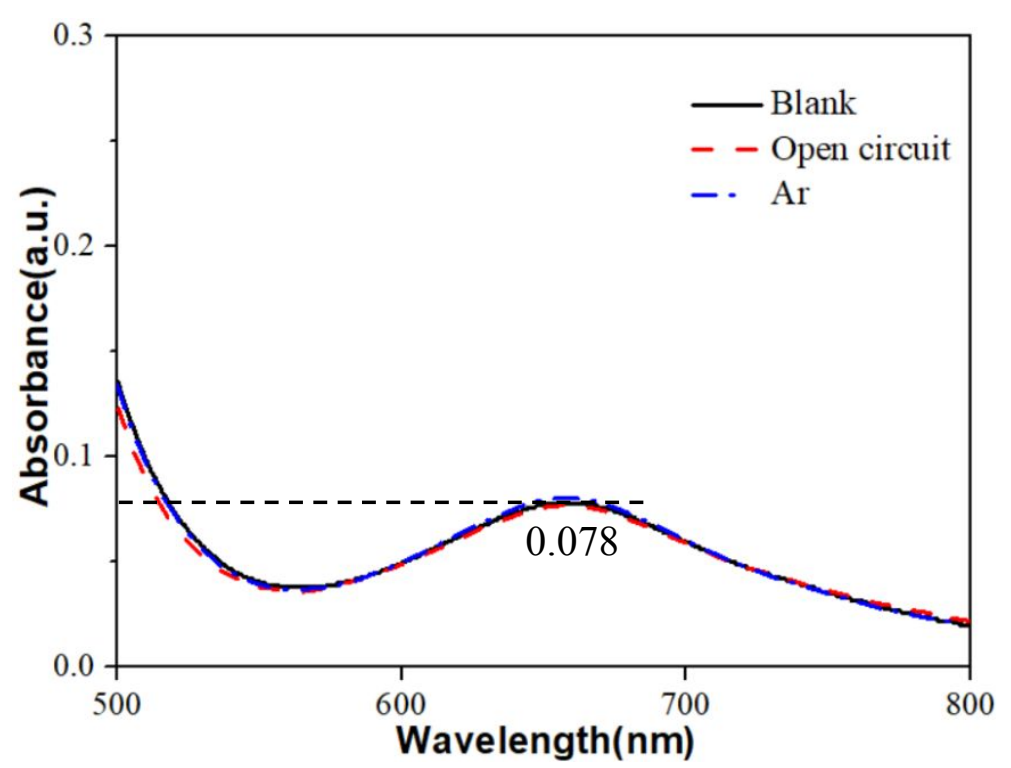

Figure S9. UV-Vis spectra of the electrolytes for blank ground, supplying $\mathrm{N}_{2}$ for $2 \mathrm{~h}$ with no applied voltage and supplying Ar for $2 \mathrm{~h}$ electrolysis at $-0.50 \mathrm{~V}$.

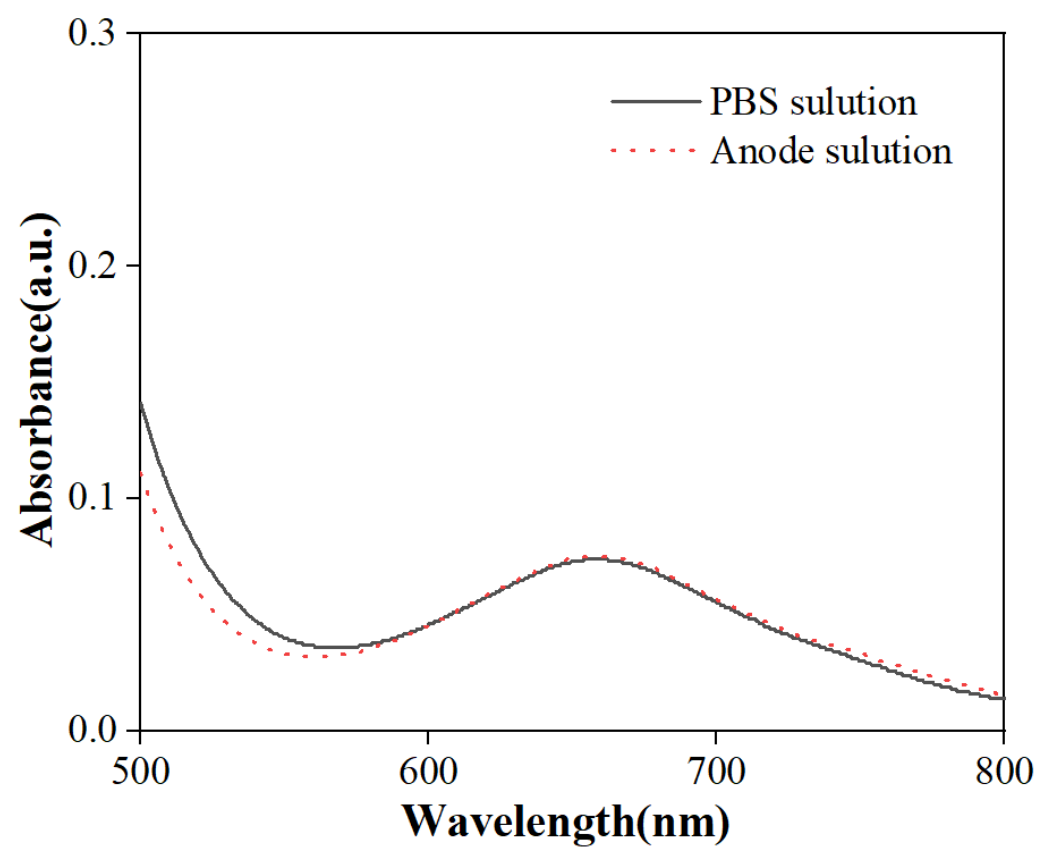

Figure S10. UV-Vis spectra of the PBS solution and anode electrolyte after NRR. 


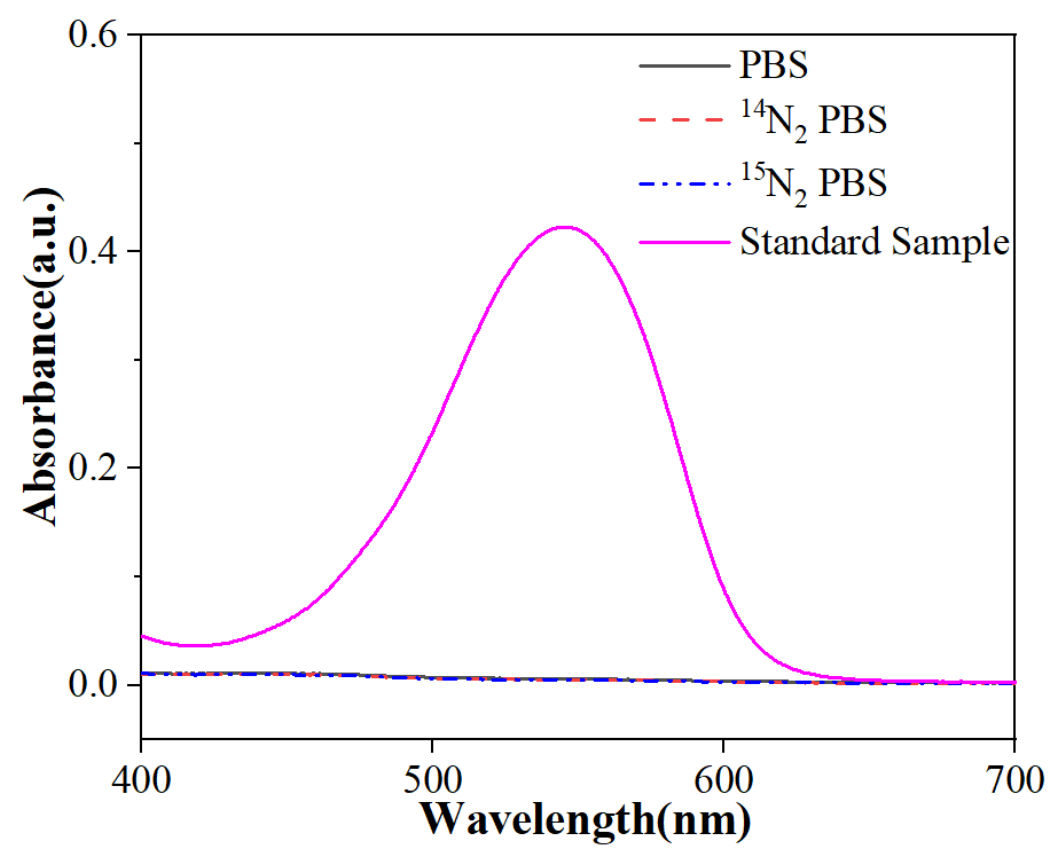

Figure S11. UV-vis adsorption spectra of nitrite detection

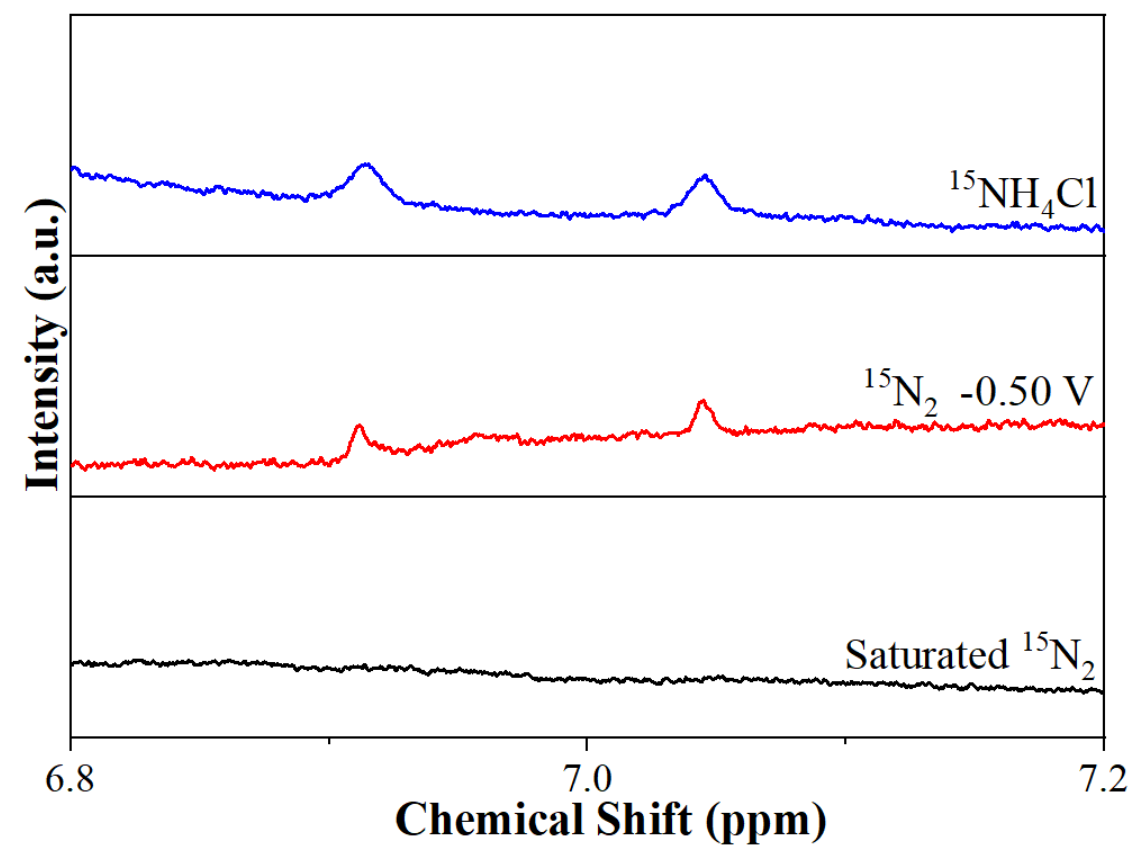

Figure S12. The ${ }^{1} \mathrm{H}$ NMR spectra of ${ }^{15} \mathrm{NH}_{4} \mathrm{Cl}$, NRR test under ${ }^{15} \mathrm{~N}_{2}$ at $-0.50 \mathrm{~V}$ and saturated ${ }^{15} \mathrm{~N}_{2}$ solution. 


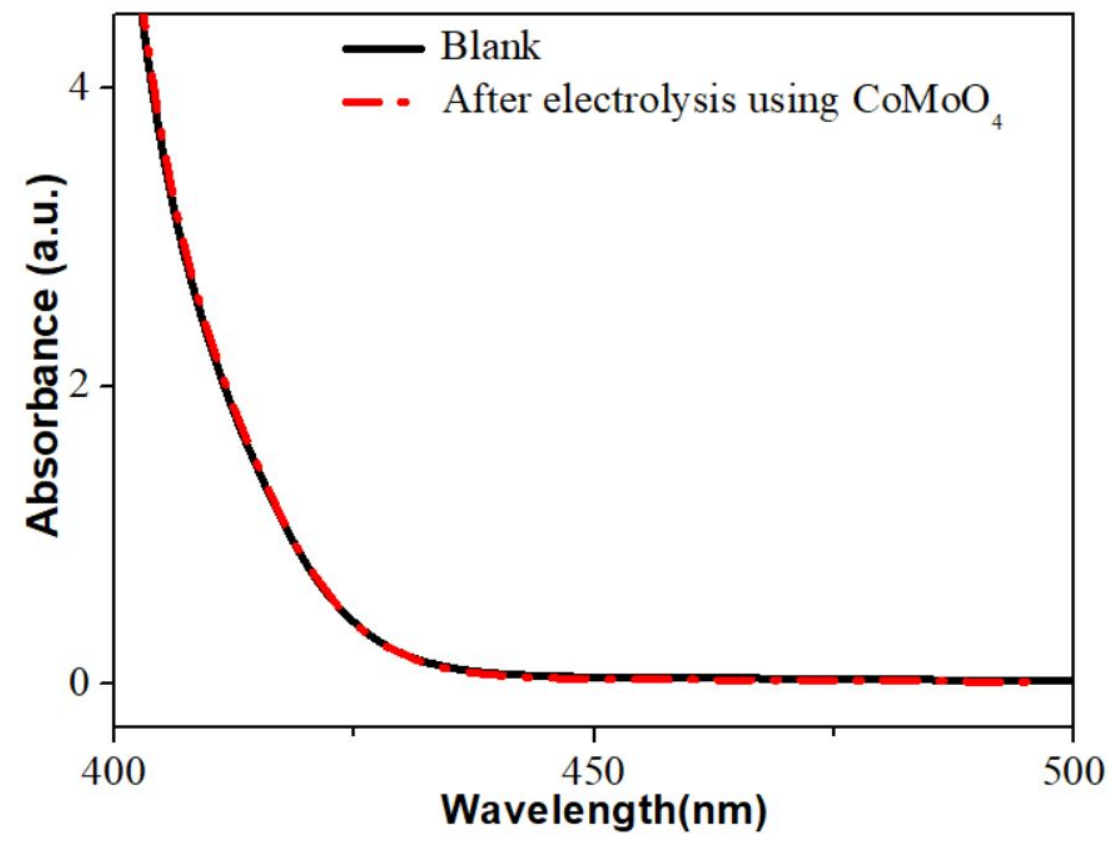

Figure S13. UV-Vis spectrum of $\mathrm{N}_{2} \mathrm{H}_{4}$ detection before and after $2 \mathrm{~h}$ electrolysis using amorphous $\mathrm{CoMoO}_{4}$ sphere at $-0.50 \mathrm{~V}$.

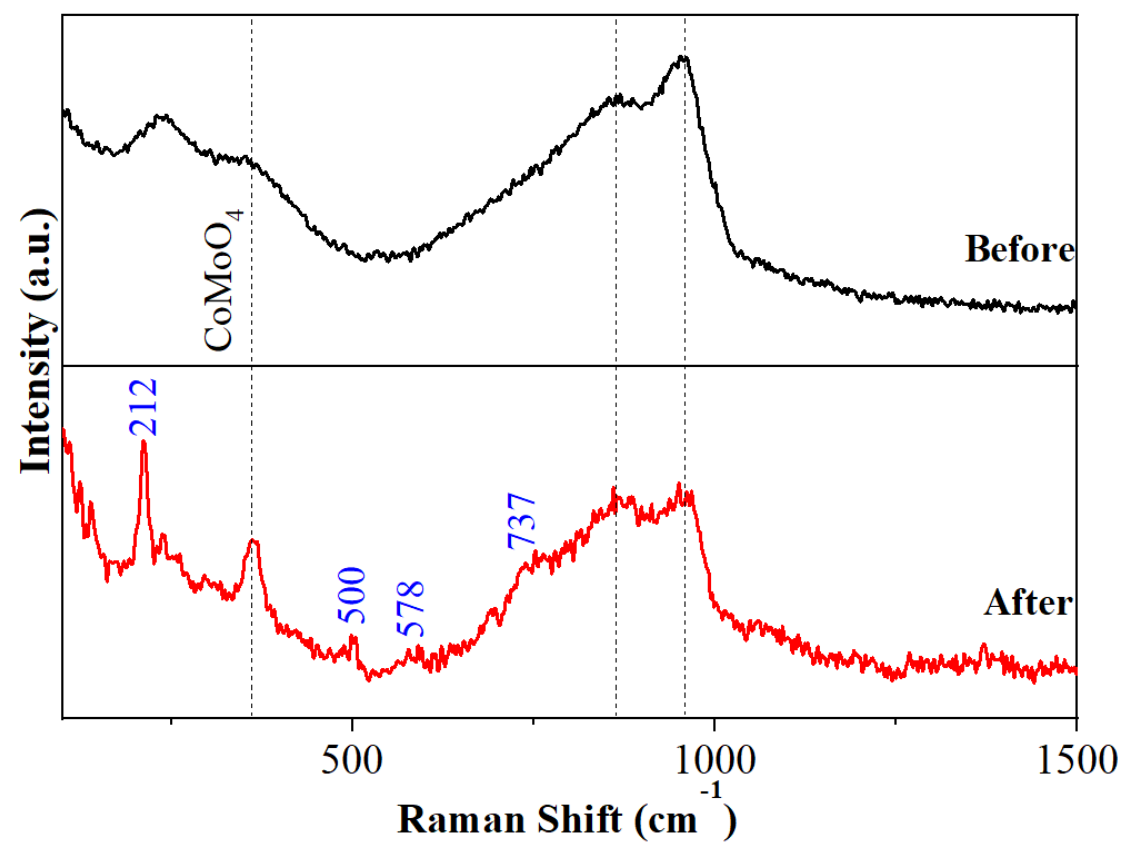

Figure S14. The Raman spectra of samples before and after stability test. 
Table S1. Comparison of the electrocatalytic NRR performance of amorphous $\mathrm{CoMoO}_{4}$ with other Mo-based and oxides electrocatalysts.

\begin{tabular}{|c|c|c|c|c|}
\hline Catalyst & Electrolyte & $\mathrm{V}_{\mathrm{NH} 3}\left(\mathrm{ug} \mathrm{h}^{-1} \mathrm{mg}_{\mathrm{cat}^{-1}}\right)$ & $\mathrm{FE}(\%)$ & Ref. \\
\hline $\mathrm{CoMoO}_{4}$ & $0.1 \mathrm{M}$ PBS & 30.2 & 3.8 & This work \\
\hline $\mathrm{O}-\mathrm{MoC}$ & $\begin{array}{l}0.1 \mathrm{mM} \mathrm{HCl}^{+} \\
0.5 \mathrm{M} \mathrm{Li}_{2} \mathrm{SO}_{4}\end{array}$ & 22.5 & 25.1 & $\begin{array}{l}\text { ACS Appl. Mater. Interfaces } \\
2019,11,31869-31877\end{array}$ \\
\hline $\mathrm{PdCu} / \mathrm{rGO}$ & $0.1 \mathrm{~m} \mathrm{KOH}$ & 2.8 & 3 & $\begin{array}{l}\text { Adv. Energy Mater. 2018, 8, } \\
1800124\end{array}$ \\
\hline $\mathrm{NbO}_{2}$ & $0.05 \mathrm{~m} \mathrm{H}_{2} \mathrm{SO}_{4}$ & 11.6 & 32 & $\begin{array}{lll}\text { Small } & \text { Methods } & 2018, \\
1800386 & & \\
\end{array}$ \\
\hline $\mathrm{LaCrO}_{3}$ & $0.1 \mathrm{M} \mathrm{Na}_{2} \mathrm{SO}_{4}$ & 24.8 & 15 & ChemCatChem 2019, 11, 1-6 \\
\hline $\mathrm{LaFeO}_{3}$ & $\begin{array}{ccc}\mathrm{M} & \mathrm{KOH} \\
80^{\circ} \mathrm{C} & \\
\end{array}$ & 13.46 & 1.99 & $\begin{array}{l}\text { Ind. Eng. Chem. Res. 2019, } \\
\text { 58, 8935-8939 }\end{array}$ \\
\hline $\mathrm{LiMn}_{2} \mathrm{O}_{4}$ & $0.1 \mathrm{M} \mathrm{HCl}$ & 15.83 & 0.72 & $\begin{array}{l}\text { Inorg. Chem. 2019, 58, } \\
\text { 9597-9601 }\end{array}$ \\
\hline $\mathrm{Bi}_{2} \mathrm{MoO}_{6}$ & $0.1 \mathrm{M} \mathrm{HCl}$ & 20.46 & 8.17 & $\begin{array}{l}\text { ACS Sustainable Chem. Eng. } \\
\text { 2019, 7, 12692-12696 }\end{array}$ \\
\hline $\mathrm{Fe}_{2}\left(\mathrm{MoO}_{4}\right)_{3}$ & $0.1 \mathrm{M} \mathrm{Na}_{2} \mathrm{SO}_{4}$ & 18.16 & 9.1 & $\begin{array}{l}\text { ASC Sunstainable Chem. } \\
\text { Eng. } 7 \text { (2019) 12692-12696. }\end{array}$ \\
\hline $\mathrm{MoS}_{2}$ & $0.1 \mathrm{M} \mathrm{Na}_{2} \mathrm{SO}_{4}$ & 29.28 & 8.34 & $\begin{array}{l}\text { Adv. Energy Mater. 2018, } \\
1801357\end{array}$ \\
\hline $\mathrm{Mn}_{3} \mathrm{O}_{4}$ & $0.1 \mathrm{M} \mathrm{Na}_{2} \mathrm{SO}_{4}$ & 11.6 & 3 & Small 2018, 1803111 \\
\hline $\mathrm{CeO}_{2}$ & $0.1 \mathrm{M} \mathrm{Na}_{2} \mathrm{SO}_{4}$ & 16.4 & 3.7 & $\begin{array}{l}\text { ACS Sustainable Chem. Eng. } \\
2019,7,2889-2893\end{array}$ \\
\hline $\mathrm{NiO} / \mathrm{G}$ & $0.1 \mathrm{M} \mathrm{Na}_{2} \mathrm{SO}_{4}$ & 18.6 & 7.8 & $\begin{array}{l}\text { ACS Appl. Energy Mater. } \\
\text { 2019, 2, 2288-2295 }\end{array}$ \\
\hline $\mathrm{Fe}_{2} \mathrm{O}_{3}$ & $0.1 \mathrm{M} \mathrm{Na}_{2} \mathrm{SO}_{4}$ & 15.9 & 0.94 & $\begin{array}{l}\text { ChemCatChem 2018, } 10, \\
4530-4535\end{array}$ \\
\hline $\begin{array}{l}\text { V-doped } \\
\mathrm{TiO}_{2} \\
\end{array}$ & $0.5 \mathrm{M} \mathrm{LiClO}_{4}$ & 17.73 & 15.9 & $\begin{array}{lll}\text { Small Methods 2019, } & \text { 20 } \\
1900356 & & \\
\end{array}$ \\
\hline$\beta-\mathrm{Bi}_{2} \mathrm{O}_{3}$ & $0.1 \mathrm{M} \mathrm{Na}_{2} \mathrm{SO}_{4}$ & 19.92 & 4.3 & $\begin{array}{l}\text { ChemCatChem 2019, 11, } \\
\text { 1884-1888 }\end{array}$ \\
\hline $\mathrm{Au} / \mathrm{CoO}_{\mathrm{x}}$ & $0.05 \mathrm{M} \mathrm{H}_{2} \mathrm{SO}_{4}$ & 15.1 & 19 & $\begin{array}{l}\text { Angew. Chem. Int. Ed. 2019, } \\
58,2-8\end{array}$ \\
\hline $\mathrm{F}-\mathrm{SnO}_{2} / \mathrm{CC}$ & $0.1 \mathrm{M} \mathrm{Na}_{2} \mathrm{SO}_{4}$ & 19.3 & 8.6 & $\begin{array}{l}\text { Inorg. Chem. 2019, 58, } \\
\text { 10424-10431 }\end{array}$ \\
\hline
\end{tabular}

